\title{
Lepton angular distribution of $W$ boson productions
}

\author{
Yang Lyu $\odot,{ }^{1,2}$ Wen-Chen Chang $\odot,{ }^{3}$ Randall Evan McClellan, ${ }^{1,4}$ Jen-Chieh Peng, ${ }^{1}$ and Oleg Teryaev ${ }^{5}$ \\ ${ }^{1}$ Department of Physics, University of Illinois at Urbana-Champaign, Urbana, Illinois 61801, USA \\ ${ }^{2}$ Department of Physics, University of California at Berkeley, Berkeley, California 94720, USA \\ ${ }^{3}$ Institute of Physics, Academia Sinica, Taipei 11529, Taiwan \\ ${ }^{4}$ Department of Physical Sciences, Pensacola State College, Pensacola, Florida 32504, USA \\ ${ }^{5}$ Bogoliubov Laboratory of Theoretical Physics, JINR, 141980 Dubna, Russia
}

(Received 5 October 2020; accepted 21 January 2021; published 15 February 2021)

\begin{abstract}
The lepton angular distribution coefficients $A_{i}$ for $Z$ boson production in $p p$ and $\bar{p} p$ collisions have been measured at the LHC and the Tevatron. A recent study showed that many features of the measured angular distribution coefficients, including the transverse momentum $\left(q_{T}\right)$ and rapidity dependencies and the violation of the Lam-Tung relation, can be well described using an intuitive geometric approach. In this paper, we extend this geometric approach to describe the angular distribution coefficients for $W$ boson produced in $\bar{p} p$ collisions at the Tevatron. We first compare the data with a perturbative QCD calculation at $\mathcal{O}\left(\alpha_{s}^{2}\right)$. We then show that the data and QCD calculations can be well described with the geometric approach. Implications for future studies at the LHC energy are also discussed.
\end{abstract}

DOI: 10.1103/PhysRevD.103.034011

\section{INTRODUCTION}

Dilepton production in hadron-hadron collision has been studied extensively following the pioneering experiment performed in 1970 [1]. The mechanism for dilepton production involves a quark annihilating with an antiquark, forming a vector boson $\left(\gamma^{*}, W\right.$, or $Z$ ), which subsequently decays into a pair of leptons. In the original Drell-Yan model [2], the vector boson was predicted to be transversely polarized, leading to an azimuthally symmetric $1+\cos ^{2} \theta$ lepton angular distribution with respect to the beam axis. This prediction agreed well with early fixedtarget dilepton production data where the transverse momentum $\left(q_{T}\right)$ of the dilepton is low [3].

The azimuthal symmetry for the lepton angular distribution no longer holds for nonzero value of $q_{T}$, and a general expression for the lepton angular distribution is given as [4]

$\frac{d \sigma}{d \Omega} \propto 1+\lambda \cos ^{2} \theta+\mu \sin 2 \theta \cos \phi+\frac{\nu}{2} \sin ^{2} \theta \cos 2 \phi$,

where $\theta$ and $\phi$ are the lepton polar and azimuthal angles in the dilepton rest frame. In the original Drell-Yan model [2], $\lambda=1$ and $\mu=\nu=0$. However, the intrinsic transverse

Published by the American Physical Society under the terms of the Creative Commons Attribution 4.0 International license. Further distribution of this work must maintain attribution to the author(s) and the published article's title, journal citation, and DOI. Funded by SCOAP ${ }^{3}$. momenta of partons and QCD effects can result in nonzero values for $\nu$ and $\mu$, while $\lambda$ can also deviate from unity. It was pointed out by Lam and Tung [4] that the amount of deviation of $\lambda$ from 1 is twice the value of $\nu$, namely, $1-\lambda=2 \nu$. This so-called Lam-Tung relation was shown to be insensitive to corrections from leading-order QCD processes [4].

The Lam-Tung relation was found to be significantly violated in pion-induced Drell-Yan experiments [5-8]. Many theoretical models [9-11] were proposed to explain this violation. In particular, Boer [11] suggested that a novel transverse-momentum dependent (TMD) parton distribution, the Boer-Mulders function [12], can give rise to a $\cos 2 \phi$ azimuthal angular modulation, resulting in a violation of the Lam-Tung relation. This not only explained the observed violation of the Lam-Tung relation but also allowed the first extraction of the Boer-Mulders functions from pion and proton induced Drell-Yan data $[11,13]$.

Recent high-statistics measurements of the lepton angular distribution coefficients in $Z$ boson production over a broad range of $q_{T}$ in $p p$ collision at the LHC by the CMS [14] and ATLAS [15] experiments revealed a clear violation of the Lam-Tung relation. Since TMD effects are only relevant at the low $q_{T}$ region, the results from LHC showed that sources other than the Boer-Mulders functions are responsible for the violations of the Lam-Tung relation at high $q_{T}$. Indeed, the fixed-order QCD calculations can account for the LHC data rather well [16].

In Ref. [17], the lepton angular distribution in $Z$ boson production was described using an intuitive geometric approach. Both the violation of the Lam-Tung relation 
and the observed $q_{T}$ dependence of $\lambda$ and $\nu$ could be well described by this approach. A subsequent paper [18] showed that this approach could explain both the $q_{T}$ and the rapidity dependencies of the lepton angular distributions. Several recent papers have also addressed various aspects of lepton angular distributions in $Z$ boson production [19-22] and the Drell-Yan process [23,24].

In addition to the lepton angular distribution data for $Z$ boson production in $\bar{p} p$ [25] and $p p$ [14,15] collisions, there are also $W$ boson production data in $\bar{p} p$ collision reported by the CDF Collaboration [26]. The important roles of the lepton angular distribution in understanding the mechanisms for $W$ and $Z$ boson production at the $\bar{p} p$ collisions at the Tevatron were pointed out in Refs. $[27,28]$. Unlike the $Z$ boson production where both $l^{-}$and $l^{+}$decay products are detected, only the charged lepton from $W$ boson decay is measured. Consequently, different experimental uncertainties are encountered in the measurements of lepton angular distributions in $W$ versus $Z$ boson production. Another important difference is that $W$ and $Z$ boson productions involve different parity-violating couplings. Therefore, it is instructive to compare the lepton angular distribution of $W$ production with that of $Z$ production. In this paper, we extend our previous geometric approach of interpreting the lepton angular distribution for $Z$ boson production to $W$ boson production.

This paper is organized as follows. In Sec. II, we briefly describe the geometric approach and present the implications of this approach on the lepton angular distribution coefficients of $W$ production. In Sec. III, we compare the $\mathrm{CDF}$ data on the angular coefficients of $W$ production with a perturbative QCD calculation. We then show in Sec. IV that the geometric approach can provide qualitative agreement with the QCD calculation and the CDF data. We also discuss possible future measurements at LHC on the angular coefficients of $W$ production. We conclude in Sec. V.

\section{GEOMETRIC APPROACH FOR LEPTON ANGULAR DISTRIBUTION COEFFICIENTS}

In hadron-hadron collision, the angular distribution of charged leptons in the $W^{ \pm}$rest frame is given by the CDF Collaboration [26] as

$$
\begin{aligned}
\frac{d \sigma}{d \Omega} \propto & \left(1+\cos ^{2} \theta\right)+\frac{A_{0}}{2}\left(1-3 \cos ^{2} \theta\right)+A_{1} \sin 2 \theta \cos \phi \\
& +\frac{A_{2}}{2} \sin ^{2} \theta \cos 2 \phi+A_{3} \sin \theta \cos \phi+A_{4} \cos \theta \\
& +A_{5} \sin ^{2} \theta \sin 2 \phi+A_{6} \sin 2 \theta \sin \phi \\
& +A_{7} \sin \theta \sin \phi
\end{aligned}
$$

where $\theta$ and $\phi$ are the polar and azimuthal angles of charged lepton in the rest frame of $W$. Comparing Eq. (2) with Eq. (1), we obtain

$$
\lambda=\frac{2-3 A_{0}}{2+A_{0}}, \quad \mu=\frac{2 A_{1}}{2+A_{0}}, \quad \nu=\frac{2 A_{2}}{2+A_{0}},
$$

and the Lam-Tung relation, $1-\lambda=2 \nu$, becomes $A_{0}=A_{2}$.

To shed some light on the meaning of the angular distribution coefficients $A_{i}$ in Eq. (2), we define three different planes in the rest frame of the $W$ boson, as shown in Fig. 1. These planes are (1) the hadron plane formed by the two colliding hadrons' momenta $\vec{p}_{B}$ and $\vec{p}_{T}$. For the Collins-Soper (C-S) frame [29], the $\hat{z}$ and $\hat{x}$ axes lie in the hadron plane where $\hat{z}$ bisects, with angle $\beta$, the two hadron momentum vectors, $\vec{p}_{B}$ and $-\vec{p}_{T}$. (2) The quark plane formed by $\hat{z}$ and the axis $\hat{z}^{\prime}$, along which a pair of quark and antiquark collide collinearly to produce a $W$ boson at rest. The polar and azimuthal angles of $\hat{z}^{\prime}$ are defined as $\theta_{1}$ and $\phi_{1}$, respectively, in the C-S frame. (3) The lepton plane defined by the momentum vector of the charged lepton $(l)$ and the $\hat{z}$ axis. It is worth noting that the definitions of these three planes and angles are completely general and independent of the specific reaction mechanism for producing the $W$ boson.

While Eq. (2) can be derived using the technique of contracting the leptonic and hadronic tensors [28,29], it is instructive to derive Eq. (2) using a different approach [17]. In the rest frame of $W$, the charged lepton angular distribution has a very simple form when it is expressed with respect to the $\hat{z}^{\prime}$ axis, namely,

$$
\frac{d \sigma}{d \Omega} \propto 1+a \cos \theta_{0}+\cos ^{2} \theta_{0}
$$

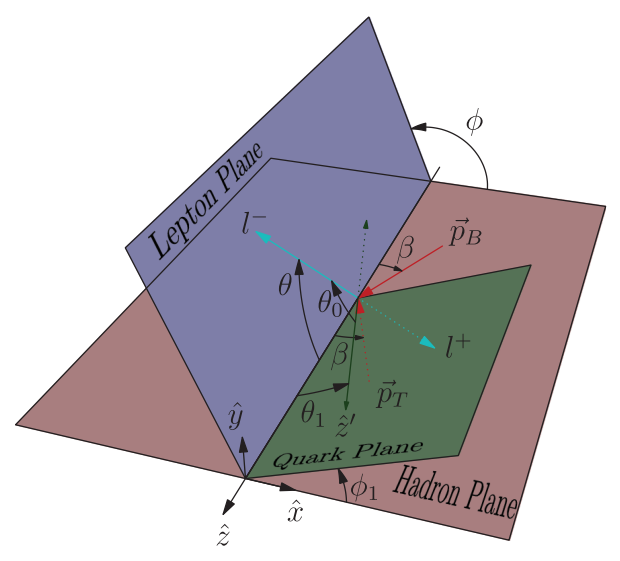

FIG. 1. Definition of the C-S frame and various planes in the rest frame of $W$ boson. The hadron plane is formed by $\vec{P}_{B}$ and $\vec{P}_{T}$, the momentum vectors of the colliding hadrons $B$ and $T$. The $\hat{x}$ and $\hat{z}$ axes of the $\mathrm{C}-\mathrm{S}$ frame both lie in the hadron plane with $\hat{z}$ axis bisecting the angle between $\vec{P}_{B}$ and $-\vec{P}_{T}$ vectors with angle $\beta$. The quark $(q)$ and antiquark $(\bar{q})$ collide head-on with equal momenta to form the $W$ boson at rest, while the quark momentum unit vector $\hat{z}^{\prime}$ and the $\hat{z}$ axis form the quark plane. The polar and azimuthal angles of $\hat{z}^{\prime}$ in the $\mathrm{C}$-S frame are $\theta_{1}$ and $\phi_{1}$. The $l$ and $\nu$ leptons are emitted back-to-back from $W$ with $\theta$ and $\phi$ specifying the polar and azimuthal angles of the charged lepton $l$. 
where $\theta_{0}$ is the polar angle of $l$ with respect to the quark momentum as shown in Fig. 1. The forward-backward asymmetry parameter, $a$, originates from the parityviolating coupling to the $W$ boson. For the Drell-Yan process involving the electromagnetic coupling to a virtual photon, parity is conserved and $a=0$. For $Z$ boson production, $a=2 A_{f} A_{f^{\prime}}$, where $A_{f}$ is a function of the vector $C_{V}^{f}$ and axial-vector $C_{A}^{f}$ couplings for $Z$ boson to fermion $f$, as discussed in Ref. [18]. As Eq. (2) is expressed in terms of the angles $\theta$ and $\phi$, one can substitute the following trigonometric relation:

$$
\cos \theta_{0}=\cos \theta \cos \theta_{1}+\sin \theta \sin \theta_{1} \cos \left(\phi-\phi_{1}\right)
$$

into Eq. (4) to obtain

$$
\begin{aligned}
\frac{d \sigma}{d \Omega} \propto & \left(1+\cos ^{2} \theta\right)+\frac{\sin ^{2} \theta_{1}}{2}\left(1-3 \cos ^{2} \theta\right)+\left(\frac{1}{2} \sin 2 \theta_{1} \cos \phi_{1}\right) \sin 2 \theta \cos \phi+\left(\frac{1}{2} \sin ^{2} \theta_{1} \cos 2 \phi_{1}\right) \sin ^{2} \theta \cos 2 \phi \\
& +\left(a \sin \theta_{1} \cos \phi_{1}\right) \sin \theta \cos \phi+\left(a \cos \theta_{1}\right) \cos \theta+\left(\frac{1}{2} \sin ^{2} \theta_{1} \sin 2 \phi_{1}\right) \sin ^{2} \theta \sin 2 \phi+\left(\frac{1}{2} \sin 2 \theta_{1} \sin \phi_{1}\right) \sin 2 \theta \sin \phi \\
& +\left(a \sin \theta_{1} \sin \phi_{1}\right) \sin \theta \sin \phi,
\end{aligned}
$$

which contains all angular terms in Eq. (2). Comparing Eq. (2) with Eq. (6), we find that all lepton angular distribution coefficients, $A_{i}$, can be expressed in terms of $\theta_{1}, \phi_{1}$, and $a$ as follows:

$$
\begin{aligned}
& A_{0}=\left\langle\sin ^{2} \theta_{1}\right\rangle \quad A_{1}=\left\langle\frac{1}{2} \sin 2 \theta_{1} \cos \phi_{1}\right\rangle \\
& A_{2}=\left\langle\sin ^{2} \theta_{1} \cos 2 \phi_{1}\right\rangle \quad A_{3}=\left\langle a \sin \theta_{1} \cos \phi_{1}\right\rangle \\
& A_{4}=\left\langle a \cos \theta_{1}\right\rangle \quad A_{5}=\left\langle\frac{1}{2} \sin ^{2} \theta_{1} \sin 2 \phi_{1}\right\rangle \\
& A_{6}=\left\langle\frac{1}{2} \sin 2 \theta_{1} \sin \phi_{1}\right\rangle \quad A_{7}=\left\langle a \sin \theta_{1} \sin \phi_{1}\right\rangle .
\end{aligned}
$$

The brackets indicate that the measured coefficients are obtained by averaging over all events. In this way, the lepton angular distribution coefficients could be related to the polar and azimuthal angles, $\theta_{1}$ and $\phi_{1}$, of the quark axis in the $W$ rest frame.

One difference between $W$ and $Z$ boson productions is that $W$ boson production maximally violates parity. The $V-A$ coupling for the $W$ boson implies a $\left(1+\cos \theta_{0}\right)^{2}$ or $\left(1-\cos \theta_{0}\right)^{2}$ lepton angular distribution for $W^{+}$ and $W^{-}$productions in Eq. (4). Hence, $|a|=2$, and Eq. (7) implies that the ranges of the angular distribution coefficients are

$$
\begin{array}{rr}
0 \leq A_{0} \leq 1, & -1 \leq A_{2} \leq 1 \\
-2 \leq A_{3} \leq 2, & -2 \leq A_{4} \leq 2,
\end{array}
$$

where we only consider $A_{0}, A_{2}, A_{3}, A_{4}$, which were measured by the CDF Collaboration. Equation (7) also shows that $A_{0} \geq A_{2}$. Therefore, when the Lam-Tung relation, $A_{0}=A_{2}$, is violated, $A_{2}$ can only be smaller, not greater, than $A_{0}$ [21].
We emphasize that the expressions of Eqs. (6)-(8) are completely general, independent of the choice of the reference frame. The exact values of the polar angles $\left(\theta, \theta_{1}\right)$ and azimuthal angles $\left(\phi, \phi_{1}\right)$ do depend, in general, on the choice of the reference frame. There are many different choices for the $W$ boson rest frame in the literature, including the Collins-Soper frame [29], the GottfriedJackson frame [30], the U-channel frame [4], the helicity frame, and the Mustraal frame [31], corresponding to different choices for the orientations of the axes.

It is possible to find the values of $\theta_{1}$ and $\phi_{1}$ for certain specific $W$ boson production processes [17,18]. Consider first a $\mathcal{O}\left(\alpha_{s}\right)$ process, $q \bar{q} \rightarrow W G$, in which a quark from one hadron annihilates with an antiquark from another hadron to form a $W$ boson. A hard gluon $G$ is emitted from either the quark or the antiquark, resulting in a nonzero transverse momentum for the $W$. It is easy to see that in the C-S frame, $\theta_{1}$ must be identical to the angle $\beta$ in Fig. 1 [18]. Emission of a gluon from one of the colliding partons cannot change the momentum of the other parton, which continues to move along the $\vec{p}_{B}$ or $\vec{p}_{T}$ direction. Hence, the $q \bar{q}$ collision axis ( $\hat{z}^{\prime}$ in Fig. 1) is along the $\vec{p}_{B}$ or $\vec{p}_{T}$ direction, making an angle $\beta$ with respect to the $\hat{z}$ axis in the $\mathrm{C}-\mathrm{S}$ frame. It is straightforward to obtain [18]

$$
\sin ^{2} \theta_{1}=\sin ^{2} \beta=q_{T}^{2} /\left(Q^{2}+q_{T}^{2}\right)
$$

where $q_{T}$ and $Q$ are the transverse momentum and mass of the $W$, respectively. Since the quark plane and the hadron plane both contain $\hat{z}$ and $\vec{p}_{B}$ (or $\vec{p}_{T}$ ), these two planes coincide and $\phi_{1}$ must vanish for this process.

For the $q G \rightarrow q^{\prime} W$ Compton process, the value of $\theta_{1}$ was found $[18,32]$ to be given approximately as

$$
\sin ^{2} \theta_{1}=5 q_{T}^{2} /\left(Q^{2}+5 q_{T}^{2}\right)
$$


while $\phi_{1}$ remains zero. It is interesting to note that at $\mathcal{O}\left(\alpha_{s}\right)$, Eq. (7) shows that the Lam-Tung relation, $A_{0}=A_{2}$, is satisfied since $\phi_{1}=0$. At $\mathcal{O}\left(\alpha_{s}^{2}\right)$ or higher, the quark plane is in general different from the hadron plane due to the emission of more than one jet $[18,22]$. Hence, $\phi_{1} \neq 0$ and the Lam-Tung relation will be violated.

\section{COMPARISON BETWEEN THE CDF DATA AND PERTURBATIVE QCD CALCULATION}

The CDF Collaboration reported the measurement of the $A_{2}$ and $A_{3}$ angular coefficients of the $W$ boson production in $\bar{p} p$ collision at $\sqrt{s}=1.8 \mathrm{TeV}$ [26]. From the detection of the charged lepton momentum from the $W \rightarrow e \nu$ and $W \rightarrow \mu \nu$ decays and the missing transverse energy $\mathbb{E}_{T}$, the azimuthal angle $\phi$ of the charged lepton in the C-S frame is measured. However, the polar angle $\theta$ of the charged lepton cannot be uniquely determined due to a twofold ambiguity resulting from the unknown longitudinal momentum of the neutrino. The $W$ boson events satisfy the requirements

$$
\begin{aligned}
E_{T}^{e}\left(P_{T}^{\mu}\right) & \geq 20 \mathrm{GeV} \quad \mathscr{E}_{T}>20 \mathrm{GeV} \\
\left|\eta^{e, \mu}\right| & \leq 1 \quad 15<q_{T}^{W}<105 \mathrm{GeV},
\end{aligned}
$$

where $\eta$ is the pseudorapidity of the charged lepton.

From the measurement of the azimuthal angle of the charged lepton in the C-S frame from the $W \rightarrow e \nu$ and $W \rightarrow \mu \nu$ decays, the angular coefficients $A_{2}$ and $A_{3}$ were extracted. With much reduced sensitivity, the coefficients $A_{2}$ and $A_{4}$ were also measured. Figure 2 shows the CDF data on $A_{0}, A_{2}, A_{3}$, and $A_{4}$ versus the transverse momentum $q_{T}$ of the $W$ boson. Both the statistical and total (statistical plus systematic) uncertainties are shown for $A_{2}$ and $A_{3}$. As the statistical uncertainties for $A_{0}$ and $A_{4}$ are large, no estimates for their systematic uncertainties were provided by the CDF Collaboration.

We first compare the CDF results with perturbative QCD calculation at $\mathcal{O}\left(\alpha_{s}^{2}\right)$. For this calculation, we utilize the DYNNLO code [33] version 1.5 [34], which provides the differential cross sections for the Drell-Yan process and $W / Z$ boson production. The CT14NNLO parton distribution functions were used for the proton and antiproton in this calculation. From the calculated $d \sigma / d \Omega$ differential angular distribution, the $A_{i}$ angular coefficients can be evaluated by taking the appropriate moments, namely [28],

$$
\begin{array}{ll}
A_{0}=4-10\left\langle\cos ^{2} \theta\right\rangle & A_{2}=10\left\langle\sin ^{2} \theta \cos 2 \phi\right\rangle \\
A_{3}=4\langle\sin \theta \cos \phi\rangle & A_{4}=4\langle\cos \theta\rangle,
\end{array}
$$

where $\langle f(\theta, \phi)\rangle$ denotes the moment of $f(\theta, \phi)$, i.e.,

$$
\langle f(\theta, \phi)\rangle=\frac{\int f(\theta, \phi) \frac{d \sigma}{d \Omega} d \Omega}{\int \frac{d \sigma}{d \Omega} d \Omega} .
$$
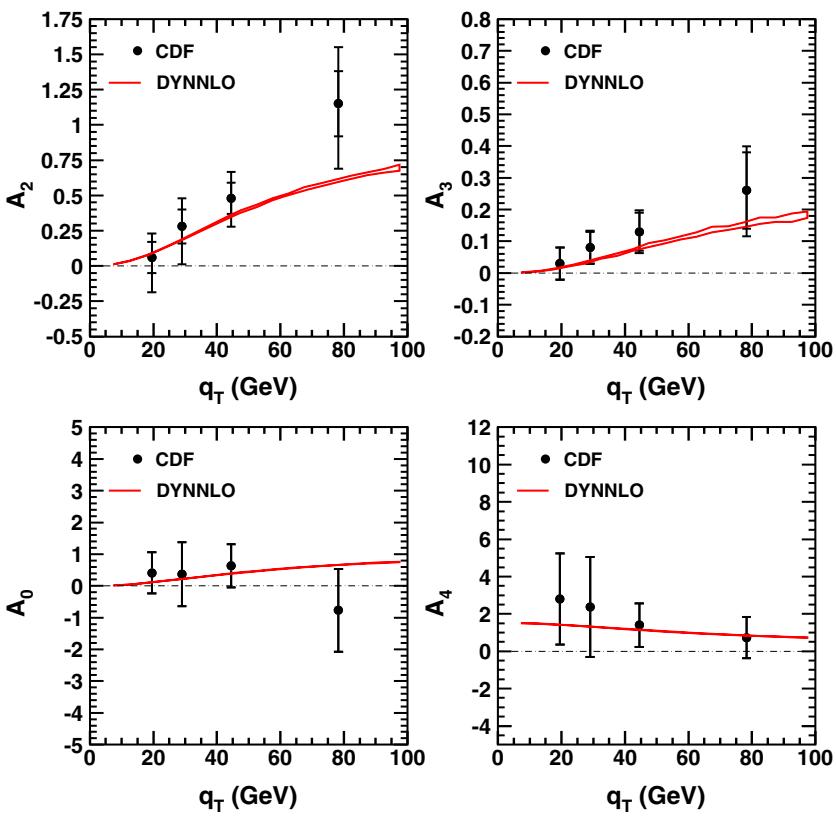

FIG. 2. Comparison between the CDF $W$ boson angular coefficient data [26] with $\mathcal{O}\left(\alpha_{s}^{2}\right)$ QCD calculation. $q_{T}$ is the transverse momentum of the $W$ boson. The coefficients $A_{2}$ and $A_{3}$ display both the statistical uncertainties (inner error bars) and the total uncertainties (outer error bars). For $A_{0}$ and $A_{4}$, only statistical error bars are provided by CDF. The $\mathcal{O}\left(\alpha_{s}^{2}\right)$ QCD calculation, shown as the solid curves, utilized the DYNNLO code and the CT14NNLO PDFs for proton and antiproton.

Equation (12) is obtained by using the orthogonality property of the various angular distribution terms in Eq. (2). The results of the calculation for $A_{0}, A_{2}, A_{3}, A_{4}$ are shown as solid curves in Fig. 2. The finite vertical widths of the curves reflect the variations when using other two PDF sets, NNPDF31nnlo and MMHT2014nnlo, for the calculation.

We note some qualitative features of the QCD calculation. As $q_{T} \rightarrow 0$, Eqs. (9) and (10) show that $\theta_{1}=0$. Equation (7) requires that all $A_{i}$ except $A_{4}$ vanish when $\theta_{1}=0$. This is confirmed by the QCD calculation shown in Fig. 2. Moreover, the upper and lower bounds listed in Eq. (8) are satisfied by the QCD calculation. The agreement between the calculation and the CDF data is quite good. We note that the present results are also in good agreement with an earlier QCD calculation performed by members of the CDF Collaboration [35].

\section{INTERPRETATION OF THE ANGULAR COEFFICIENTS WITH THE GEOMETRIC APPROACH}

While the $\mathcal{O}\left(\alpha_{s}^{2}\right)$ QCD calculation can describe the angular coefficients of $W$ production very well as shown in Fig. 2, it is instructive to examine how well the intuitive geometric approach discussed in Sec. II can reproduce the main features of the data. In the earlier studies of the $Z$ 
boson production $[17,18]$, the high statistics of the LHC data made it possible to use the data to constrain some parameters in the geometric approach. Unfortunately, the large uncertainty for the $W$ boson production data from CDF greatly limits the sensitivity of using the data to test the geometric approach. Therefore, we use instead the $\mathcal{O}\left(\alpha_{s}^{2}\right)$ QCD results to check whether the geometric approach can adequately describe the angular coefficients for $W$ boson production.

We start with the $A_{0}$ angular coefficient. Equation (7) shows that $A_{0}$ is given by the values of $\sin ^{2} \theta_{1}$ averaged over the different processes. At $\mathcal{O}\left(\alpha_{s}\right)$, Eqs. (9) and (10) give the $q_{T}$ dependence of $\sin ^{2} \theta_{1}$ for the quark-antiquark annihilation and the quark-gluon Compton process, respectively. The dotted and dot-dashed curves in Fig. 3 correspond to Eqs. (9) and (10), respectively. Note that the $q G$ process alone overestimates $A_{0}$, while $q \bar{q}$ underestimates it. As the $q \bar{q}$ annihilation and the $q G$ Compton processes involve different initial states, they contribute incoherently to the $W$ production. The dashed curve in Fig. 3 is obtained with the following expression:

$$
A_{0}=f \frac{q_{T}^{2}}{Q^{2}+q_{T}^{2}}+(1-f) \frac{5 q_{T}^{2}}{Q^{2}+5 q_{T}^{2}},
$$

where $f$ represents the fraction of $q \bar{q}$ process, and $1-f$ is the fraction of the $q G$ process. The best fit to the QCD calculation gives $f=0.610 \pm 0.002$, which is consistent with the expectation that the $q \bar{q}$ annihilation process dominates the $q G$ process in $p \bar{p}$ collision. The excellent agreement between the geometric approach and the QCD

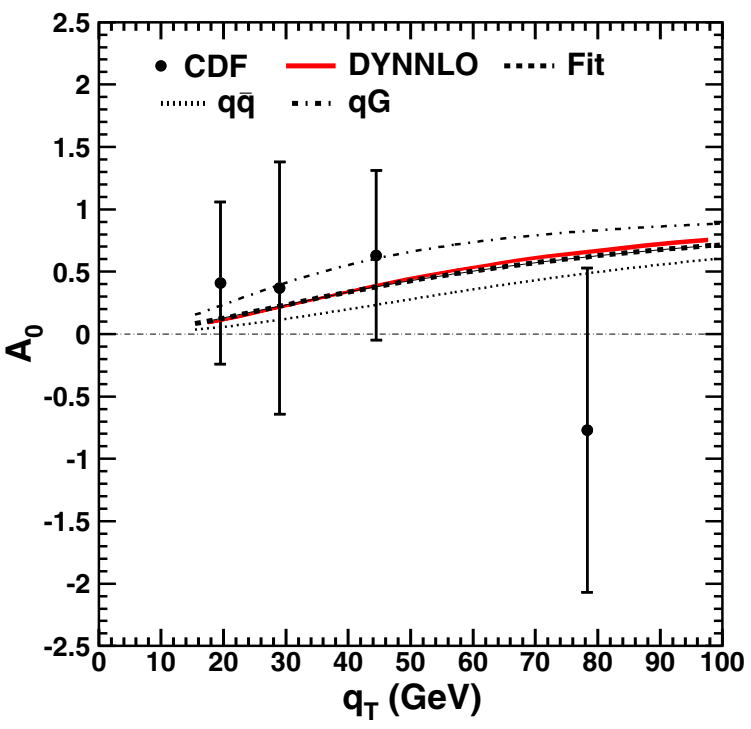

FIG. 3. Comparison between the $\mathcal{O}\left(\alpha_{s}^{2}\right)$ QCD (solid curve) and the geometric approach (dashed curve) for the calculations of $A_{0}$. The dotted and dot-dashed curves correspond to the contribution from the $q \bar{q}$ and $q G$ subprocess, respectively, in the geometric approach. The CDF data points [26] are also displayed. calculation suggests that Eqs. (9) and (10) are capable of reproducing the main features of the QCD calculation for $A_{0}$.

We next consider the $A_{2}$ angular coefficient. If LamTung relation is satisfied, then $A_{0}=A_{2}$. Figure 4 compares the $\mathcal{O}\left(\alpha_{s}^{2}\right)$ QCD calculation for $A_{2}$ (solid curve) with the result of $A_{0}$ from the geometric approach (dotted curve) obtained with Eq. (14). While the agreement is reasonable, the QCD calculation is consistently below the dotted curve. This indicates that the Lam-Tung relation, $A_{0}=A_{2}$, is violated. In the geometric approach, this implies that the angle $\phi_{1}$ is nonzero, which leads to a smaller $A_{2}$ than $A_{0}$, as shown in Eq. (7). A nonzero $\phi_{1}$ implies that the quark and hadron planes are not coplanar. This non-coplanarity is caused by higher-order QCD processes at $\mathcal{O}\left(\alpha_{s}^{2}\right)$ or higher, in which multiple partons accompany the $W$ boson in the final state. To account for the nonzero $\phi_{1}$ angle, we use the following expression:

$A_{2}=\left(f \frac{q_{T}^{2}}{Q^{2}+q_{T}^{2}}+(1-f) \frac{5 q_{T}^{2}}{Q^{2}+5 q_{T}^{2}}\right) \cos 2 \phi_{1}$.

The dashed curve in Fig. 4 corresponds to Eq. (15) with $f=0.610$ obtained from the $A_{0}$ data discussed above and the best-fit value for $\cos 2 \phi_{1}=0.905 \pm 0.004$. This corresponds to an average non-coplanarity angle, $\phi_{1}$, of $12.6^{\circ}$. The improved agreement between the geometric model and QCD calculation using this nonzero $\phi_{1}$ angle indicates the effects of $\mathcal{O}\left(\alpha_{s}^{2}\right)$ or higher, which allows the hadron plane to deviate from the quark plane. An analogous situation was found for $Z$ boson production and discussed in [18].

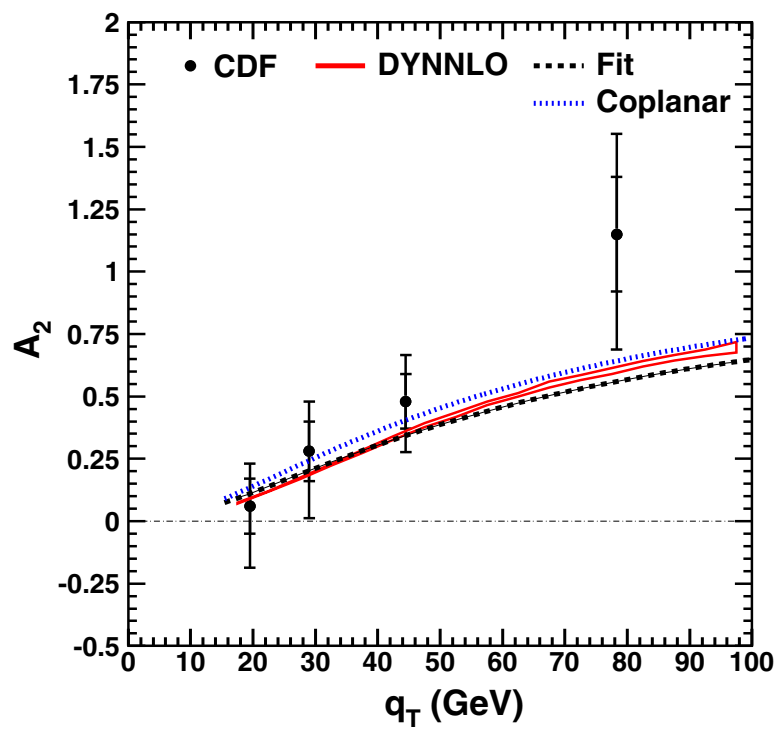

FIG. 4. Comparison between the $\mathcal{O}\left(\alpha_{s}^{2}\right)$ QCD (solid curve) and the geometric approach (dashed curve) for the calculations of $A_{2}$. The dotted curve corresponds to the calculation of the geometric model when the non-coplanarity angle $\phi_{1}$ is set at zero. The CDF data points [26] are also displayed. 
The good agreement between the simple calculation using Eq. (15) and the sophisticated QCD calculation again illustrates the useful insight provided by the geometric approach for understanding the angular coefficient in $W$ boson production.

We consider next the parity-violating angular coefficient $A_{4}$. From Eq. (7), the expression for $A_{4}$ is the product of the forward-backward asymmetry parameter $a= \pm 2$ and $\cos \theta_{1}$. Since the expressions for $\sin ^{2} \theta_{1}$ are given by Eqs. (9) and (10) for the $q \bar{q}$ and $q G$ processes, we use the following expression for $A_{4}$ :

$A_{4}=2 r_{4}\left(f \frac{Q}{\left(Q^{2}+q_{T}^{2}\right)^{\frac{1}{2}}}+(1-f) \frac{Q}{\left(Q^{2}+5 q_{T}^{2}\right)^{\frac{1}{2}}}\right)$,

where the factor of 2 on the right-hand side signifies the magnitude of the forward-backward asymmetry, $|a|=2$. The parameter $r_{4}$, which has a magnitude less than 1 , is to account for the fact that the sign of $a$ is either positive or negative, depending on whether it is $q \bar{q} \rightarrow W$ or $\bar{q} q \rightarrow W$ process, as discussed in Ref. [18] for the analogous $Z$ boson production. Depending on the relative weight of these two contributions, governed by the partonic distributions of quarks and antiquarks in the proton and antiproton, the magnitude of $A_{4}$ is expected to be reduced from the partial cancellation effect. The parameter $r_{4}$ accounts for such a partial cancellation effect. The dashed curve in Fig. 5 shows that Eq. (16), using the best-fit value of $r_{4}=0.738 \pm$ 0.002 , is in excellent agreement with the QCD calculation. The large uncertainty of the $A_{4}$ measurement from CDF prevents a conclusive comparison between the QCD and geometric model calculation with the data. In fact, the constraint $A_{4}<2$ from Eq. (8), marginally violated by the

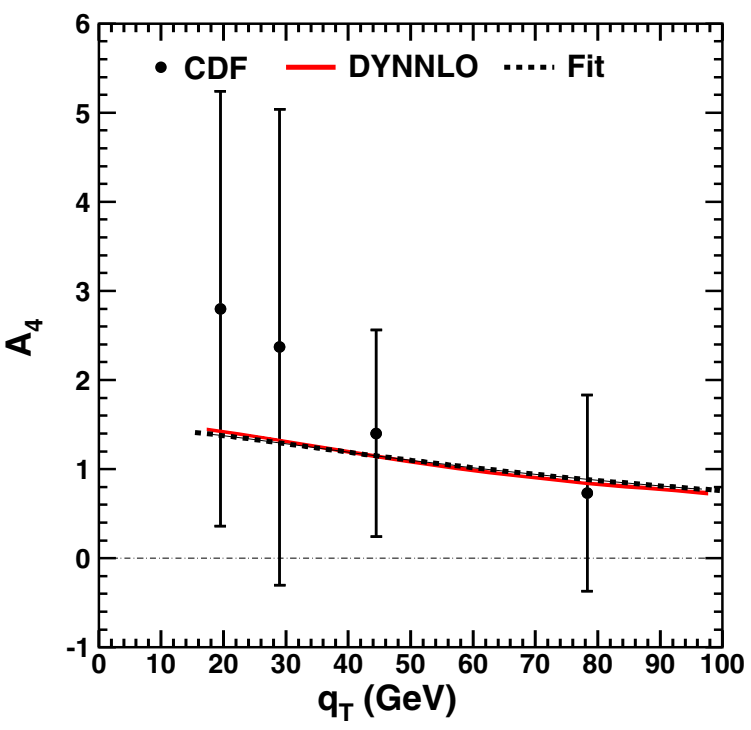

FIG. 5. Comparison between the $\mathcal{O}\left(\alpha_{s}^{2}\right)$ QCD (solid curve) and the geometric model (dashed curve) for $A_{4}$. The CDF data points [26] are also displayed. central values of the data points at the lowest two $q_{T}$ values, is satisfied by the QCD calculation. The fact that the simple calculation of Eq. (16) can describe the $\mathcal{O}\left(\alpha_{s}^{2}\right)$ QCD calculation very well again indicates the adequacy of the simple geometric model in understanding the main features of the angular coefficients in $W$ boson production.

We turn to the $A_{3}$ coefficient next. As shown in Eq. (7), $A_{3}$ involves all three quantities, $\theta_{1}, \phi_{1}$, and $a$. The partial cancellation effects discussed for $A_{4}$ are also expected for $A_{3}$. From Eqs. (7), (9), and (10), we use the following expression for $A_{4}$ in the geometric model:

$A_{3}=2 r_{3}\left(f \frac{q_{T}}{\left(Q^{2}+q_{T}^{2}\right)^{\frac{1}{2}}}+(1-f) \frac{\sqrt{5} q_{T}}{\left(Q^{2}+5 q_{T}^{2}\right)^{\frac{1}{2}}}\right) \cos \phi_{1}$,

where the factor of 2 is again the forward-backward asymmetry parameter for $W$ boson production, and $\phi_{1}=$ $12.6^{\circ}$ was obtained in the previous analysis of $A_{2}$. Since $A_{3}$ is an odd function under the $\phi_{1} \leftrightarrow \pi-\phi_{1}$ exchange, a large cancellation effect is expected [18]. Therefore, we expect the value of the reduction factor, $r_{3}$, to be small. The dashed curve in Fig. 6 is the best fit to the $\mathcal{O}\left(\alpha_{s}\right)$ QCD calculation using Eq. (17). Indeed, the value of $r_{3}$ is found to be quite small, $r_{3}=0.0540 \pm 0.001$, confirming a very large cancellation effect. Moreover, the agreement between the calculations of the geometric model and the $\mathcal{O}\left(\alpha_{s}^{2}\right)$ QCD is not very good. This suggests that the simple assumption that $r_{4}$ is independent of $q_{T}$ is no longer a good assumption in the presence of strong cancellation effects. Nevertheless, the general feature that $A_{3}$ increases with $q_{T}$ can still be described by the geometric model.

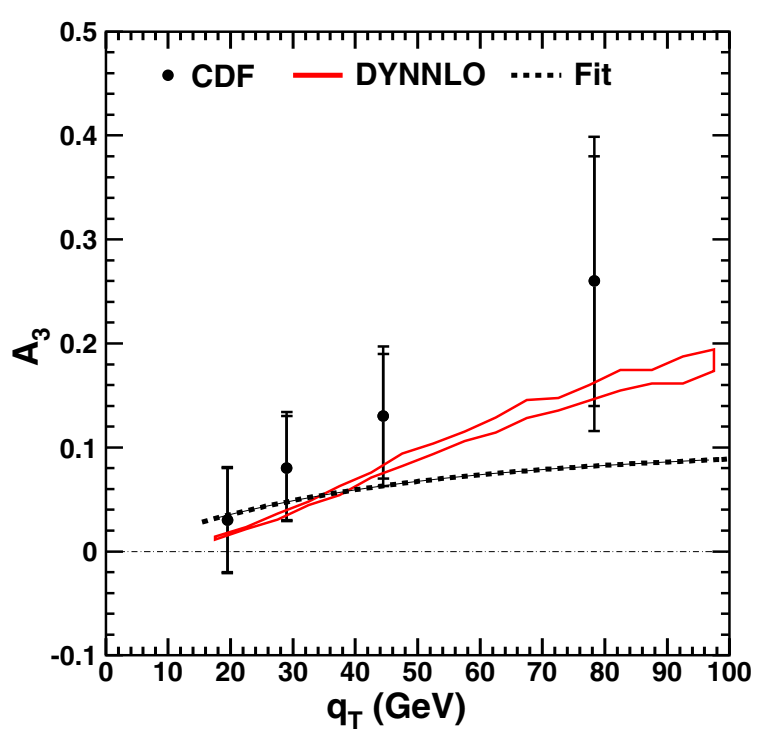

FIG. 6. Comparison between the $\mathcal{O}\left(\alpha_{s}^{2}\right)$ QCD (solid curve) and the geometric model (dashed curve) for $A_{3}$. The CDF data points [26] are also displayed. 
We conclude this section by discussing the prospect for collecting and analyzing $W$ boson angular distribution data at the LHC. The high luminosity and high center-of-mass energy at the LHC allow a precise measurement of $W$ and $Z$ boson production. Indeed, the lepton angular distribution data for $Z$ production reported by CMS and ATLAS have demonstrated a much higher precision and broader $q_{T}$ coverage than at Tevatron. It is still challenging to measure $W$ boson angular distribution due to the missing neutrinos. As a result, only the $W$ polarization parameters, $f_{L}, f_{R}$, and $f_{0}$ in the helicity frame have been measured $[36,37]$ so far. Nevertheless, as shown by the CDF Collaboration, at least the $A_{2}$ and $A_{3}$ coefficients in the C-S frame could be measured with adequate precision even at Tevatron. It is anticipated that LHC could at least allow a very precise measurement of $A_{2}$ and $A_{3}$ coefficients for $W$ production. As discussed in a recent paper [22], the $A_{2}$ coefficient is expected to have very different $q_{T}$ distributions for $Z$ boson plus single jet or multiple jets. Similar expectation also holds for $W$ plus jets production at the LHC. In particular, the $A_{2}$ values for $W$ plus multiple-jets events are expected to be smaller than for the $W$ plus single-jet events. This is due to the nonzero values of $\phi_{1}$ for a multiple-jets events, while a single-jet $W$ production event must have $\phi_{1}=0$. Equation (7) then implies that $A_{2}$ for multiple-jet events must be smaller than that for single-jet events. This prediction remains to be tested by the LHC experiments.

We note that a recent paper suggests the possibility of reconstructing the $W$ decay angular distribution in the Mustraal frame [31]. From an analysis of the Monte Carlo data at the LHC energy, it was shown that the Mustraal frame has the interesting property that all angular coefficients except $A_{4}$ have vanishing values. An inspection of Eq. (4) shows that the $z$ axis of the Mustraal frame coincides with the $\hat{z}^{\prime}$ axis. Thus far, all existing data on $W$ and $Z$ boson production from LHC are analyzed in either the C-S frame or the helicity frame. Future analysis of these data in the Mustraal frame would be of considerable interest.

\section{SUMMARY AND CONCLUSIONS}

In this paper, we have extended the geometric approach to describe the angular distribution coefficients of $W$ boson production at the CDF. In this geometric approach, first discussed in [17], all of the eight lepton angular distribution coefficients can be expressed as trigonometric expressions involving three quantities: $a, \theta_{1}$, and $\phi_{1}$. The quantity $\theta_{1}$ refers to the polar angle of the collinear quark-antiquark axis in the $W$ boson rest frame, $\phi_{1}$, the non-coplanarity angle between the plane formed by the two hadrons, and the lepton plane containing the leptons from the $W$ decay. The parity-violating parameter $a$ has a magnitude of 2 for $W$ production. These trigonometric expressions lead to a set of upper and lower bounds for the various angular coefficients, as well as some relationships between these angular coefficients. In particular, the Lam-Tung relation refers to the equality of the $A_{0}$ and $A_{2}$ coefficients when the non-coplanarity angle $\phi_{1}$ vanishes. The violation of the Lam-Tung relation is then attributed to a nonzero $\phi_{1}$ angle, resulting in $A_{0}>A_{2}$. For the $q \bar{q}$ annihilation and $q G$ Compton processes at $\mathcal{O}\left(\alpha_{s}\right), \phi_{1}$ vanishes, and the LamTung relation is valid. For processes at $\mathcal{O}\left(\alpha_{s}^{2}\right)$ or higher, $\phi_{1}$ can be nonzero and the Lam-Tung relation will be violated.

We first compare the CDF angular coefficient data with $\mathcal{O}\left(\alpha_{s}^{2}\right)$ QCD calculation. Although the statistical precision of the CDF data is only marginal, the general features of the data are in good agreement with the QCD calculations. We then compare the QCD results with the expressions obtained from the geometric model in order to determine several parameters in this model. Good agreement between the QCD calculations and the geometric approach is obtained. We also confirm that the QCD calculations and the geometric approach satisfy the upper and lower bounds derived for the angular coefficients, as well as the inequality $A_{0}>A_{2}$. The implication of this study for $W$ production at LHC is also discussed. In particular, a high precision measurement of $A_{2}$ for $W$ plus jets events is feasible and of much interest.

We emphasize that this geometric approach is developed to provide some simple intuitive insights for understanding the angular distribution coefficients for $W$ and $Z$ boson production. It is certainly not a substitute for the rigorous perturbative QCD calculations. The good agreement between the geometric approach and the perturbative QCD calculation as well as the data is reassuring that the geometric model has some merits in understanding the main features of the lepton angular distributions, including their transverse momentum dependence and the violation of the Lam-Tung relation, in an intuitive fashion. We expect that this geometric approach can also be extended to other hard processes, including the Drell-Yan process, quarkonium production, $e^{-} e^{+}$collision, and deep-inelastic scattering.

\section{ACKNOWLEDGMENTS}

This research was supported in part by the U.S. National Science Foundation Grant No. PHYS18-22502 and the Ministry of Science and Technology of Taiwan. 
[1] J. H. Christenson, G. S. Hicks, L. M. Lederman, P. J. Limon, B. G. Pope, and E. Zavattini, Phys. Rev. Lett. 25, 1523 (1970).

[2] S. D. Drell and T. M. Yan, Phys. Rev. Lett. 25, 316 (1970); Ann. Phys. (N.Y.) 66, 578 (1971).

[3] I. R. Kenyon, Rep. Prog. Phys. 45, 1261 (1982).

[4] C. S. Lam and W. K. Tung, Phys. Rev. D 18, 2447 (1978).

[5] S. Falciano et al., Z. Phys. C 31, 513 (1986).

[6] M. Guanziroli et al., Z. Phys. C 37, 545 (1988).

[7] J. S. Conway et al., Phys. Rev. D 39, 92 (1989).

[8] J. G. Heinrich et al., Phys. Rev. D 44, 1909 (1991).

[9] A. Brandenburg, S. J. Brodsky, V. V. Khoze, and D. Müller, Phys. Rev. Lett. 73, 939 (1994).

[10] K. Eskola, P. Hoyer, M. Väntinnen, and R. Vogt, Phys. Lett. B 333, 526 (1994).

[11] D. Boer, Phys. Rev. D 60, 014012 (1999).

[12] D. Boer and P. J. Mulders, Phys. Rev. D 57, 5780 (1998).

[13] L. Y. Zhu et al., Phys. Rev. Lett. 99, 082301 (2007); 102, 182001 (2009).

[14] V. Khachatryan et al. (CMS Collaboration), Phys. Lett. B 749, 187 (2015).

[15] G. Aad et al. (ATLAS Collaboration), J. High Energy Phys. 08 (2016) 159.

[16] R. Gauld, A. Gehrmann-De Ridder, T. Gehrmann, E. W. N. Glover, and A. Huss, J. High Energy Phys. 11 (2017) 003.

[17] J. C. Peng, W. C. Chang, R. E. McClellan, and O. Teryaev, Phys. Lett. B 758, 384 (2016).

[18] W. C. Chang, R. E. McClellan, J. C. Peng, and O. Teryaev, Phys. Rev. D 96, 054020 (2017).

[19] J. C. Martens, J. P. Ralston, and J. P. Tapia Takaki, Eur. Phys. J. C 78, 5 (2018).
[20] M. Gavrilova and O. Teryaev, Phys. Rev. D 99, 076013 (2019).

[21] J. C. Peng, D. Boer, W. C. Chang, R. E. McClellan, and O. Teryaev, Phys. Lett. B 789, 356 (2019).

[22] J. C. Peng, W. C. Chang, R. E. McClellan, and O. Teryaev, Phys. Lett. B 797, 134895 (2019).

[23] M. Lambertsen and W. Vogelsang, Phys. Rev. D 93, 114013 (2016).

[24] W. C. Chang, R. E. McClellan, J. C. Peng, and O. Teryaev, Phys. Rev. D 99, 014032 (2019).

[25] T. Aaltonen et al. (CDF Collaboration), Phys. Rev. Lett. 106, 241801 (2011).

[26] D. Costa et al. (CDF Collaboration), Phys. Rev. D 73, 052002 (2006).

[27] E. Mirkes, Nucl. Phys. B387, 3 (1992).

[28] E. Mirkes and J. Ohnemus, Phys. Rev. D 50, 5692 (1994).

[29] J. C. Collins and D. E. Soper, Phys. Rev. D 16, 2219 (1977).

[30] K. Gottfried and J. D. Jackson, Nuovo Cimento 33, 309 (1964).

[31] E. Richter-Was and Z. Was, Eur. Phys. J. C 77, 111 (2017).

[32] R. L. Thews, Phys. Rev. Lett. 43, 987 (1979).

[33] S. Catani and M. Grazzini, Phys. Rev. Lett. 98, 222002 (2007); S. Catani, L. Cieri, G. Ferrera, D. de Florian, and M. Grazzini, Phys. Rev. Lett. 103, 082001 (2009).

[34] DYNNLO v1.5, http://theory.fi.infn.it/ grazzini/dy.html.

[35] J. Strologas and S. Errede, Phys. Rev. D 73, 052001 (2006).

[36] S. Chatrchyan et al. (CMS Collaboration), Phys. Rev. Lett. 107, 021802 (2011).

[37] M. Aaboud et al. (ATLAS Collaboration), Eur. Phys. J. C 79, 535 (2019). 